\title{
In vitro effects of Ala16Val manganese superoxide dismutase gene polymorphism on human white blood cells exposed to methylmercury
}

T.D. Algarve' ${ }^{1}$, F. Barbisan², E.E. Ribeiro ${ }^{3}$, M.M.M.F. Duarte ${ }^{4}$, M.F. Mânica-Cattani ${ }^{1}$, C.P. Mostardeiro ${ }^{2}$, A.F. Lenz ${ }^{1}$ and I.B.M. da Cruz ${ }^{1,2,4}$

${ }^{1}$ Programa de Pós-Graduação em Ciências Biológicas: Bioquímica Toxicológica, Universidade Federal de Santa Maria, Santa Maria, RS, Brasil

${ }^{2}$ Programa de Pós-Graduação em Farmacologia,

Universidade Federal de Santa Maria, Universidade Federal de Santa Maria, Santa Maria, RS, Brasil

${ }^{3}$ Universidade Aberta da Terceira Idade, Universidade do Estado do Amazonas, Manaus, AM, Brasil

${ }^{4}$ Laboratório de Biogenômica, Departamento de Morfologia,

Centro de Ciências da Saúde, Universidade Federal de Santa Maria,

Universidade Federal de Santa Maria, Santa Maria, RS, Brasil

Corresponding author: T.D. Algarve

E-mail: thais.algarve@gmail.com

Genet. Mol. Res. 12 (4): 5134-5144 (2013)

Received October 30, 2012

Accepted April 26, 2013

Published October 29, 2013

DOI http://dx.doi.org/10.4238/2013.October.29.7

ABSTRACT. Environmental contamination by methylmercury (MeHg) is an enormous public health problem in world regions such as Amazonia. $\mathrm{MeHg}$ toxic effects seem to be influenced by environmental and genetic factors. However, few studies have evaluated the genetic influences of $\mathrm{MeHg}$ toxicity in humans. Therefore, the aim of this study was to evaluate the genetic influence of Ala16Val manganese superoxide dismutase gene polymorphism (Ala16Val-MnSOD) on the cytotoxic effects of in vitro human leukocytes exposed to $\mathrm{MeHg}$. Subjects were selected from 100 individuals aged $26.4 \pm 7.3$ years genotyped to Ala16Val-MnSOD 
polymorphism $(\mathrm{AA}=6, \mathrm{VV}=6$, and $\mathrm{AV}=12)$ to perform in vitro testing using white blood cells (WBCs). Reactive oxygen species production was measured using 2',7'-dichlorofluorescein diacetate fluorimetric assay, and cell viability was measured using MTT assay on WBC samples from the same subjects that were both exposed and not exposed to $\mathrm{MeHg}(2.5 \mu \mathrm{M}$ for $6 \mathrm{~h})$. The results showed that AA- and VV-WBCs exposed to $\mathrm{MeHg}$ did not display increased reactive oxygen species levels compared to those in cells that were not exposed. However, AVleukocytes exposed to $\mathrm{MeHg}$ displayed increased ROS levels. Cellular viability comparison among genotypes exposed to $\mathrm{MeHg}$ showed that the viability of AA-WBCs was lower than that of VV-WBC, with mean values of $3.46 \pm 0.13$ and $3.08 \pm 0.77$ (standard error), respectively $(\mathrm{P}=$ 0.033 ), whereas heterozygous cells (AV) displayed intermediate values. This difference was likely due to the higher basal $\mathrm{H}_{2} \mathrm{O}_{2}$ production of AA-WBCs compared to that of other genotypes. These results suggest that the Ala16Val-MnSOD polymorphism has toxicogenetic effects in human cells exposed to $\mathrm{MeHg}$.

Key words: Ala16Val manganese superoxide dismutase polymorphism; Methyl mercury; Cell viability; Toxicogenetic

\section{INTRODUCTION}

Methylmercury (MeHg) is a well-known potential threat to human health owing to its capacity to cause systemic toxicity that damages the body through neurotoxic effects; motor function impairment (Clarkson and Magos, 2006); immune, kidney, and cardiovascular system dysfunction (Moszczyński et al., 1998; Rutowski et al., 1998; Virtanen et al., 2007); and genotoxicity (Grotto et al., 2009).

The process responsible for triggering $\mathrm{MeHg}$ toxicity is incompletely understood and likely involves metabolic alterations such as increased oxidative stress (Su et al., 2008) and inflammatory response (Havarinasab and Hultman, 2005). Previous investigations have described the relationship of $\mathrm{MeHg}$ to changes in the activities of antioxidant enzymes such as superoxide dismutase (SOD) (Ariza et al., 1998) and catalase (CAT) (Hussain et al., 1999). It also induces the production of reactive oxygen species (ROS), especially hydrogen peroxide $\left(\mathrm{H}_{2} \mathrm{O}_{2}\right)$ and superoxide anion radical $\left(\mathrm{O}_{2}^{-}\right)$(Lund et al., 1991; do Nascimento et al., 2008), which promotes oxidative stress and lipid peroxidation. Populations exposed to high $\mathrm{Hg}$ levels such as those that occur in Amazonian communities have displayed oxidative stress biomarkers measured in whole blood, plasma, and hair, as well as significant inverse relationships between exposure and antioxidant enzymes such as glutathione peroxidase (GPx) and CAT have clearly demonstrated an association between $\mathrm{Hg}$ exposure and oxidative stress (Grotto et al., 2010). However, the dynamic of the Hg toxicological process in human cells and tissues involving oxidative metabolism is difficult to determine owing to the influence of several environmental and gene factors.

Conversely, gene polymorphism studies related to oxidative stress triggered by $\mathrm{Hg}$ intoxication metabolism have focused mainly on glutathione (GSH)-related enzymes (Engström 
et al., 2011). However, to define genetically susceptible risk groups completely, research is also needed on the genes/proteins involved in the toxicodynamics - i.e, the mechanisms causing adverse effects in the organism (Gundacker et al., 2010). Therefore, gene polymorphism studies related to other antioxidant enzymes may improve understanding of the differences in $\mathrm{Hg}$ susceptibility among individuals.

Of particular interest is the manganese SOD enzyme (MnSOD), a primary antioxidant enzyme in the mitochondria that converts ROS into oxygen and $\mathrm{H}_{2} \mathrm{O}_{2}$ (Ferreira and Matsubara, 1997). ROS in mitochondria are produced via an oxidative phosphorylation pathway involved in energy production in the mitochondrial electron transport chain. Complexes I and III are the primary sources of $\mathrm{O}_{2}^{--}$production in mitochondria. Superoxide is released into the matrix from complex I, whereas it is released into both the matrix and the inter-membrane space by complex III (Murphy, 2009). In addition to being a major site of ROS production, mitochondria are a target for ROS and reactive nitrogen species and are compromised by severe or prolonged oxidative stress (Zorov et al., 2006). These conditions instigate a vicious cycle that amplifies mitochondrial ROS, whereby mitochondrial ROS/reactive nitrogen species cause oxidative damage to mitochondrial DNA leading to further mitochondrial dysfunction and oxidant generation (Fukai and Ushio-Fukai, 2011).

The human MnSOD enzyme gene displays a diallelic polymorphism in the mitochondrial targeting sequence (MTS) in which alanine 16 (GCT) is replaced with a valine (GTT) - the Ala16Val polymorphism (Ala16Val MnSOD). This polymorphism affects the import of MnSOD into the mitochondria by altering the conformation of its leader signal (Zelko et al., 2002). A study performed by Sutton et al. (2005), has suggested the Ala-MnSOD precursor generates $30-40 \%$ more MnSOD homotetramer than that generated by the Val-MnSOD precursor. Therefore, the Ala-MnSOD mitochondrial targeting sequence allows efficient MnSOD import into the mitochondrial matrix, whereas the Val-variant causes partial arrest of the precursor within the inner membrane and decreased formation of the active SOD tetramer in the mitochondrial matrix. The Ala16Val polymorphism results in 3 genotypes: AA, VV, and AV (Sutton et al., 2005).

Several studies, including our own investigations and those of other researchers, have suggested that the Ala16Val MnSOD polymorphism is associated with various oxidative stress-dependent pathologies. Interestingly, in some cases, the disease risk is associated with the A allele or AA genotype - for example, in prostate cancer (Taufer et al., 2005), breast cancer (Bica et al., 2009), and other cancers. Disease risk is linked to the V allele or VV genotype in diabetes microvascular complications (Tian et al., 2011), obesity (Montano et al., 2009), hypercholesterolemia (Duarte et al., 2010), and metastatic potential of breast cancer (Bica et al., 2010).

Recently, we began testing whether this polymorphism influences responses to toxic agents in the human body. The first in vitro protocol that evaluated whether cell carriers of Ala16Val MnSOD polymorphism display differential responses when exposed to ultraviolet radiation (UV) showed that human VV-lymphocyte cell cultures had lower viability, lower mitotic index, and higher lipoperoxidation levels than those of AA genotypes in both control and UV-exposed groups (dos Santos Montagner et al., 2010). Furthermore, this polymorphism has in vitro influence on antioxidant drug response (Costa et al., 2012). Therefore, we postulated that Ala16Val MnSOD polymorphism influences the viability of white blood cells (WBCs) exposed to MeHg in vitro. 


\section{MATERIAL AND METHODS}

\section{Reagents}

All of the chemicals used for the biochemical and molecular analyses in this study were purchased from Sigma (St. Louis, MO, USA), Invitrogen (Carlsbad, CA, USA), Mo Bio (Carlsbad, CA, USA), BD Diagnostics (Plymouth, DN, UK) and Cultilab Co. (Campinas, SP, BR).

\section{Subject selection}

To test MeHg cytotoxicity in human WBCs in carriers of different Ala16Val genotypes, we analyzed ROS production and cell viability. Blood was obtained from healthy adult subjects enrolled at Universidade Federal de Santa Maria in a Brazilian region with no history of $\mathrm{MeHg}$ exposure (Rio Grande do Sul). Initially, we selected 100 young, healthy people (age, $26.4 \pm 7.3$ years old) and considered the following factors for inclusion: non-smoking, not obese, no chronic use of medication or vitamin supplements, no previous cardiovascular medical history or hypertensive disorder, and no metabolic diseases or other morbidity that could affect the results. The research study described herein was approved by the ethics committee of the Universidade Federal de Santa Maria (No. 23081.015838/2011-10). All blood cell donors signed a consent form.

\section{Ala16Val MnSOD genotyping}

Blood samples were collected from the study group via Vacutainer ${ }^{\circledR}$ tubes with EDTA. Genomic DNA was isolated using a DNA Mini Purification Kit. Genotypes of Ala16Val MnSOD gene polymorphism was assessed via polymerase chain reaction and restriction fragment length polymorphism (PCR-RFLP) as described by Costa et al. (2012).

\section{In vitro treatments}

A subgroup of the initial study group was asked to donate blood again for in vitro tests because a previous study performed by Montano et al. (2009), has shown an association between the Ala16Val polymorphism, dietary behavior, and health conditions. The $8 \mathrm{~mL}$ blood samples were collected via venipuncture using tubes with heparin, which were centrifuged within $1 \mathrm{~h}$ of collection for $15 \mathrm{~min}$ at $3000 \mathrm{rpm}$. Then the WBC were collected and resuspended in PBS buffer with $2 \%$ glucose at a final concentration of $1 \times 10^{6}$ cells $/ \mathrm{mL}$. Two treatments were performed for each sample: negative control and concentrated exposure to $2.5 \mu \mathrm{M} \mathrm{MeHg}$ (Shenker et al., 2002). The samples were maintained in sterile conditions at $37^{\circ} \mathrm{C}$ for $6 \mathrm{~h}$.

\section{Cell viability assays}

Cell viability assays were performed using a 96-well plate. Cytotoxicity was evaluated using a 3-(4,5dimethylthiazol-2-yl)-2,5-diphenyltetrazolic bromide reduction assay described by Mosmann (1983), the absorbance of each well was measured at $570 \mathrm{~nm}$ (SpectraMax M2/ M2e Multi-mode Plate Reader, Molecular Devices Corporation, Sunnyvale, CA, USA). All tests were performed in triplicate for each sample. 


\section{Reactive oxidative species (ROS) WBC production}

Intracellular ROS production was detected in WBC samples using the 2',7'-dichlorofluorescein diacetate (DCFDA). The fluorescence was measured at an excitation of 485 $\mathrm{nm}$ and an emission of $520 \mathrm{~nm}$ (SpectraMax M2/M2e Multi-mode Plate Reader, Molecular Devices Corporation, Sunnyvale, CA, USA). All tests were performed in triplicate for each of the samples tested (LeBel et al., 1992; Halliwell and Whiteman, 2004).

\section{Statistical analysis}

All analyses were carried out using SPSS, version 18.0 (SPSS Inc., Chicago, IL, USA). Cytotoxicity in WBC samples from different Ala16Val MnSOD donors treated with and without $\mathrm{MeHg}$ was compared using 1-way analysis of variance followed by post hoc Tukey's test. All P values were 2-tailed. The alpha value was set to $<0.05$ to determine statistical relevance.

\section{RESULTS}

The MnSOD genotype frequencies were as follows: $\mathrm{AA}=31 \%(31), \mathrm{VV}=23 \%$, and $\mathrm{AV}=46 \%$. The calculation of possible deviation from Hardy-Weinberg equilibrium, which was used to assess the $\mathrm{c} 2$ goodness of fit, showed that the samples were in genetic equilibrium. From this sample, we selected 24 volunteers $(\mathrm{AA}=06, \mathrm{VV}=06$, and $\mathrm{AV}=$ 12) with similar lifestyle behaviors to perform the WBC in vitro exposure to avoid possible environmental interferences.

Initially, we evaluated the potential toxicity of $\mathrm{MeHg}$ treatment considering the whole of the WBC samples. We observed that after in vitro treatment, the viability of WBCs exposed to $\mathrm{MeHg}$ decreased significantly to $89.1 \pm 15.4 \%(\mathrm{P}=0.022)$. The viability was also compared among WBC samples with different Ala16Val MnSOD genotypes without MeHg treatment. After $6 \mathrm{~h}$, the WBC viability under in vitro conditions showed differences among Ala16Val MnSOD genotypes. The absorbance in the AA [3.46 \pm 0.13 standard error (SE)] and AV $(3.23 \pm 0.8 \mathrm{SE})$ groups was significantly higher than that in the VV group (3.08 \pm $0.77 \mathrm{SE} P=0.033)$, indicating that $\mathrm{VV}$ cells presented lower viability than that of AA and $\mathrm{AV}$ cells independent of $\mathrm{MeHg}$ treatment. ROS production was also higher in VV than in $\mathrm{AA}$ and $\mathrm{AV}$ control groups $(\mathrm{VV}=477.7 \pm 137.45 ; \mathrm{AA}=343.9 \pm 184.3 ; \mathrm{AV}=297.0 \pm 155.7$ $\mathrm{P}=0.0001)$.

MeHg WBC ROS and cytotoxicity were influenced by the Ala16Val MnSOD genotypes. As shown in Figure 1, in the AA-WBC samples, the ROS production was similar between the control and $\mathrm{MeHg}$ treatments $(\mathrm{P}=0.532)$. Similar results were also observed in $\mathrm{VV}-\mathrm{WBC}$ samples $(\mathrm{P}=0.516)$. However, in the heterozygous $(\mathrm{AV}-\mathrm{WBC})$, the ROS production increased $27 \%$ when exposed to $\mathrm{MeHg}(\mathrm{P}=0.037)$.

Despite the AA-WBC present similar ROS production between the control and $\mathrm{MeHg}$ treatments, cell viability decreased significantly in the presence of $\mathrm{MeHg}(\mathrm{P}=0.020)$. As seen in Figure 2, these results were not observed in the VV-WBC and AV-WBC groups. Cell viability was similar between the control and MeHg treatments $(\mathrm{P}=0.145$ and $\mathrm{P}=$ 0.759 , respectively). 


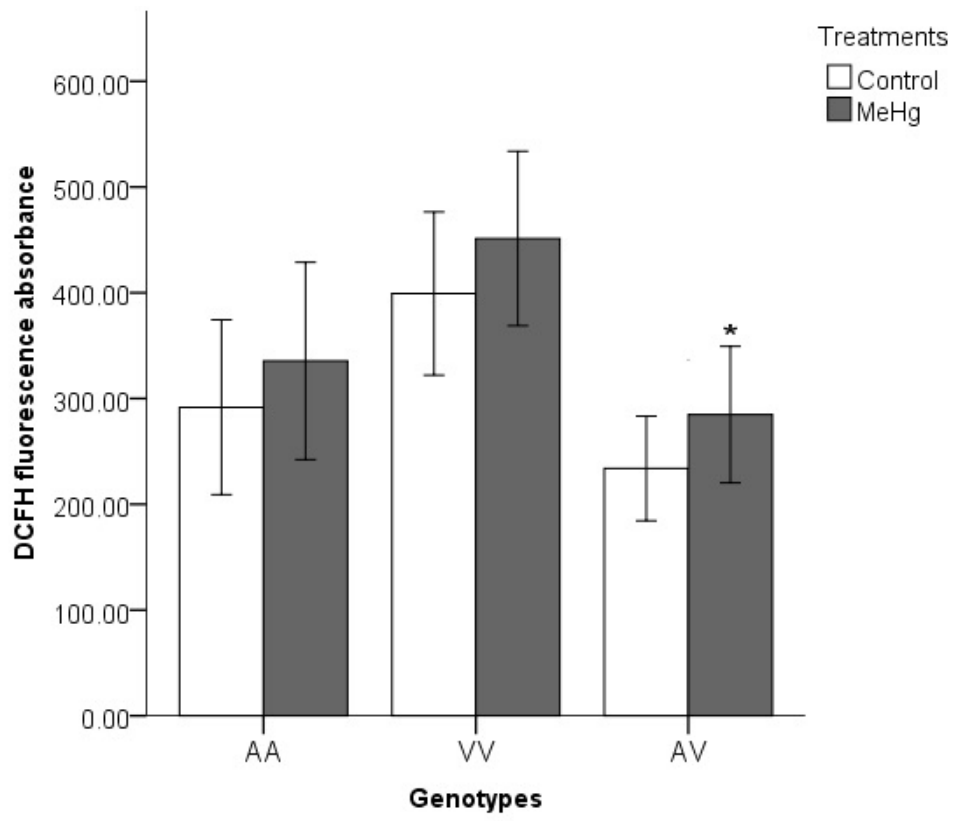

Figure 1. Reactive oxygen production (ROS) comparison among white blood cell carriers (WBC) and different Ala16Val MnSOD genotypes. The asterisk indicates a significant increase from the untreated control $(\mathrm{P}<0.05)$.

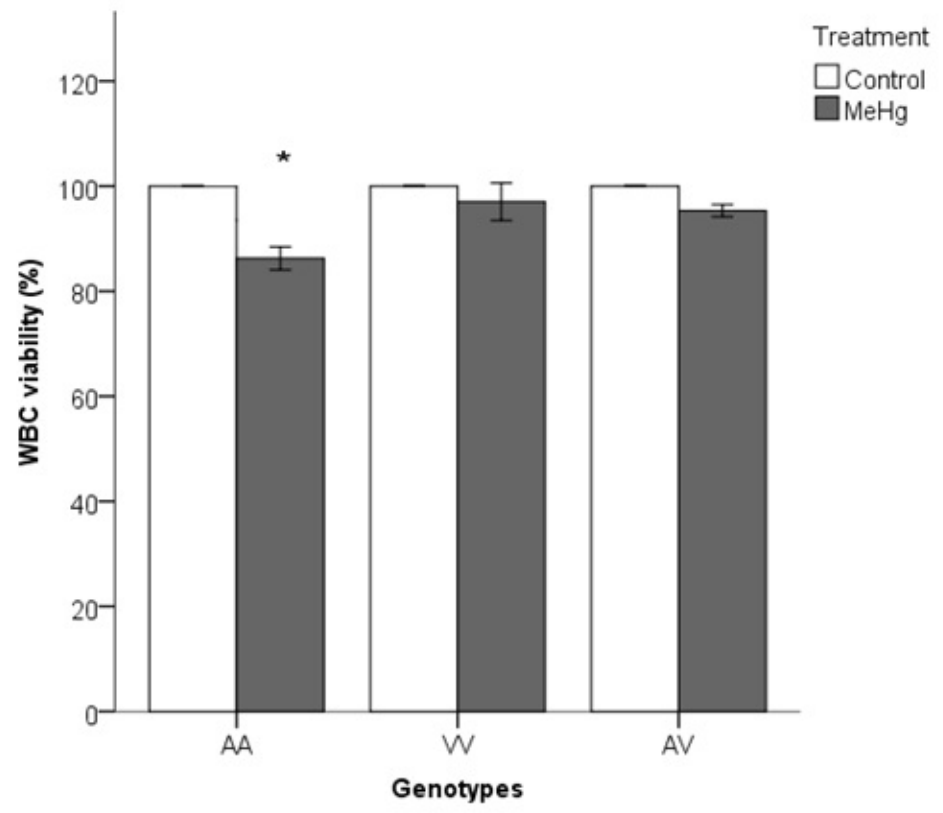

Figure 2. Viability (\% in relation to control group considered as $100 \%$ viability) between WBC with different Ala16Val MnSOD genotypes exposed to $\mathrm{MeHg}$ compound. The asterisk indicates significant decrease from the untreated control $(\mathrm{P}<0.05)$. 


\section{DISCUSSION}

In vitro $\mathrm{MeHg}$ exposure in WBCs obtained from human subjects increase ROS and decreased cell viability. The results corroborate investigations performed in animals and in vitro protocols that have shown an increase in cellular MeHg-induced oxidative stress and cytotoxicity effects. In vivo and in vitro studies have demonstrated that $\mathrm{MeHg}$-exposed systems in various species display an increase in reactive species such as $\mathrm{O}_{2}{ }^{-}, \mathrm{H}_{2} \mathrm{O}_{2}$, and nitric oxide (Farina et al., 2011). Excessive free radical formation has been implicated as a causative factor in neurotoxic damage associated with a variety of metals, including MeHg. MeHg-induced systems increase ROS formation in astrocytes as well as in the presence of superoxide. Hydroxyl scavenger antioxidant molecules attenuate MeHg-induced ROS formation (Shanker et al., 2005). Bussolaro et al. (2010) have investigated the hepatocytes of the tropical fish Hypostomus commersoni and observed that $\mathrm{MeHg}$ deregulates the redox intracellular milieu leading to cell death and oxidative stress.

The severity of toxic effects depends on the dose, duration, and timing of exposure. Humans, however, show a greatly varying susceptibility toward toxic substances even under comparable exposure situations. Several biological and non-biological factors (e.g.; age, gender, diet) can modulate individual susceptibility to toxic compounds. Gene variation plays a certain role because it constitutes various detoxifier phenotypes ranging from individuals with regular enzyme and detoxification functions to poor metabolizers with low or no enzyme activity to high metabolizers (Gundacker et al., 2010).

In humans, cytosolic GSTs catalyze intracellular conjugation reactions between GSH and various electrophilic substrates, among them Hg. These GSH conjugates can be easily effluxed from cells. Concomitant GSH depletion must be compensated by de novo synthesis (Dickinson and Forman, 2002). Thus, the interplay of all GSH system components is required for GSH affine mercury to be rapidly bound and removed from the cell. Evidence suggests that gene variants can influence mercury toxicokinetics. This genetic background can therefore potentially explain some of the individual vulnerability toward mercury toxicity (Gundacker et al., 2010).

Mercury is capable of altering the expression and activity of many genes/proteins, but functional proof of that influence is lacking. Many studies of Hg toxicokinetic and detoxification pathways have analyzed the impact of gene knockout/knockdown or a gene sequence variation (mostly single-nucleotide polymorphisms) (Gundacker et al., 2010), although none of these polymorphisms is as directly related to $\mathrm{Hg}$ toxicity as much as SODs are by ROS toxicity pathways. The major daily source of ROS in the cell is the mitochondria, in which MnSOD is located to protect against $\mathrm{O}_{2}{ }^{--}$and the Ala16Val polymorphism executes its role.

Our results were directly influenced by the Ala16Val MnSOD genotypes of WBC samples. VV-WBCs exposed to MeHg presented no increase in ROS production, nor did they display cell viability similar to that in the control group, suggesting a protective role against mercury intoxication. However, AA-WBCs displayed similar ROS production with and without $\mathrm{MeHg}$ exposition, whereas cells exposed to $\mathrm{MeHg}$ displayed decreased cell viability compared to that of the control group. The heterozygous genotype (AV) was the intermediate phenotype of AA- and VV-WBCs because it displayed an increase in ROS production but no decreased cell viability. These results may be related to $\mathrm{H}_{2} \mathrm{O}_{2}$ production, which is lower in the VV genotype compared with that in the AA genotype.

The MnSOD enzyme belongs to a set of antioxidant defense mechanisms that prevent high levels of free radicals, limiting deleterious effects to macromolecules in the cell. Because su- 
peroxide radicals are produced by nicotinamide adenine dinucleotide phosphate oxidase, xanthine oxidase, nitric oxide synthase, lipoxygenase, and mitochondrial enzymes, they must be highly controlled by the cell. A superoxide radical is converted by SOD to $\mathrm{H}_{2} \mathrm{O}_{2}$, which in turn is reduced to water by CAT, GPx, and peroxiredoxins. Thus, MnSOD (as well as others SODs) is considered a first line of defense against the toxicity of superoxide anion radicals (Fukai and Ushio-Fukai, 2011).

This enzyme is synthesized in the cytoplasm and directed to the mitochondria by a peptide signal. Therein, it is involved in dismutating superoxide radicals generated mainly by the electron transport chain from cellular respiration. The Ala16Val MnSOD polymorphism in the peptide signal region has 2 homozygous genotypes with unbalanced MnSOD activity because the AA genotype present higher efficiency of enzyme transport into the mitochondria than VV genotype. In biochemical terms, the AA genotype converts a higher quantity of superoxide radicals in $\mathrm{H}_{2} \mathrm{O}_{2}$. However, unlike SOD1, MnSOD does not show allosteric inhibition by $\mathrm{H}_{2} \mathrm{O}_{2}$ production, and the excess $\mathrm{H}_{2} \mathrm{O}_{2}$ produced by AA can undergo spontaneous conversion or react with transition metals, producing a hydroxyl radical ( $\left.{ }^{\circ} \mathrm{OH}\right)$ (Beyer and Fridovich, 1987). Because the hydroxyl radical presents high affinity to the DNA molecule causing mutations, the Ala allele or genotype AA has been associated with an increased risk for breast cancer (Taufer et al., 2005; Bica et al., 2009).

Conversely, the VV genotype does not display efficient superoxide radical dismutation in $\mathrm{H}_{2} \mathrm{O}_{2}$. The superoxide radical is extremely reactive, mainly with nitric oxide, leading to the production of the strong oxidant peroxynitrite, which has a higher affinity for the lipids of the membrane that causes lipoperoxidation. The VV genotype has been associated with carotid atherosclerosis (Kakko et al., 2003) and higher oxidized low-density lipoprotein levels in Brazilian subjects with synergic effects in type II diabetes (Gottlieb et al., 2005), obesity (Montano et al., 2009), and hypercholesterolemia (Duarte et al., 2010).

The results described herein and those of our previous studies showed that in vitro lymphocyte exposure to UV radiation produced lower toxicity in AA cells compared with that in VV cells (dos Santos Montagner et al., 2010). Therefore, we postulated that an MnSOD gene presents a dual protective characteristic dependent on toxic agents (Figure 3). This dual characteristic is similar to that observed in the association between the Ala16Val MnSOD gene polymorphism and susceptibility to non-transmissible diseases (Bica et al., 2010).

Studies supporting this hypothesis are still incipient. However, Lund et al. (1991) have observed that $\mathrm{Hg}$ compounds such as mercuric ion present mitochondrial GSH as a principal intracellular target and increase $\mathrm{H}_{2} \mathrm{O}_{2}$ approximately 4-fold in rat kidney mitochondria. The authors suggest that $\mathrm{H}_{2} \mathrm{O}_{2}$ formation by mercuric ion may lead to oxidative tissue damage, such as lipid peroxidation, which is observed in $\mathrm{Hg}$-induced nephrotoxicity.

Another question that needs to be answered is related to WBCs as cell potentially affected by $\mathrm{Hg}$ exposure. Studies using animal models have demonstrated that $\mathrm{Hg}$ affects immune function in a complex manner. This influence depends on both the species of $\mathrm{Hg}$ used and the genetic background under which exposure takes place. In genetically susceptible mouse strains, inorganic and organic $\mathrm{Hg}$ species, including $\mathrm{MeHg}$, induce autoimmunity, resulting in a lupus-like condition (Havarinasab and Hultman, 2005). Investigations suggest that humans may also be susceptible to the immunotoxic effects of $\mathrm{MeHg}$ exposure, which has been associated with an increased risk of lupus and greater severity of scleroderma (Cooper et al., 2004). Among the toxic effects of $\mathrm{MeHg}$ on polymorphonuclear leukocytes was stimulation of ROS formation, including superoxide radicals and $\mathrm{H}_{2} \mathrm{O}_{2}$ (Jansson and Harms-Ringdahl, 1993). Therefore, WBCs can be a good model for in vitro evaluation of the potential influence 
of human genetic polymorphisms in responses to Hg contamination. Based on these results, complementary investigations may improve understanding of the biochemical influence of MnSOD modulation in cellular MeHg toxicity.

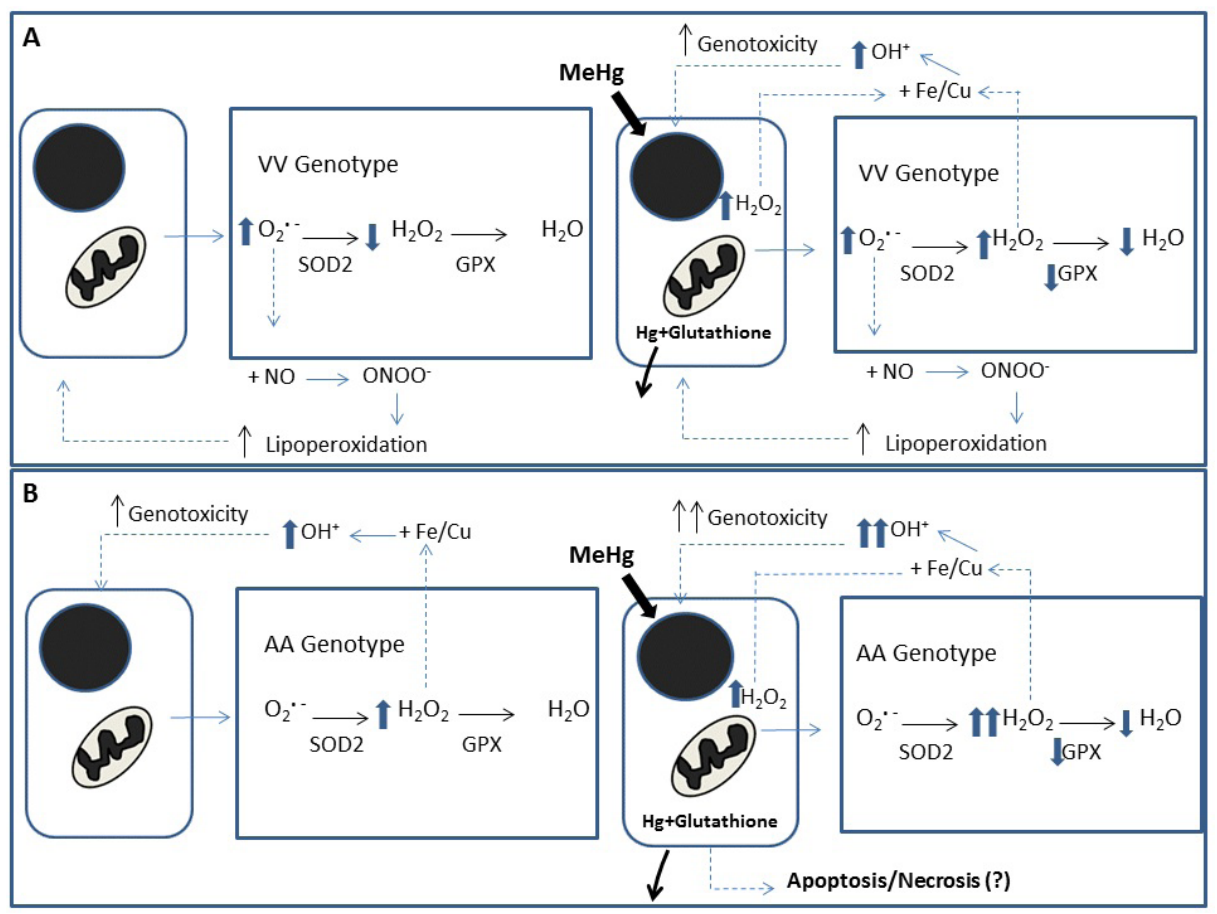

Figure 3. Synthesis of the potential protective role of human VV genotypes of Ala16Val MnSOD gene polymorphism on toxic effect caused by $\mathrm{MeHg}$ acute exposition. A. VV genotype metabolizes smaller rates of $\mathrm{O}_{2}{ }^{--}$than $\mathrm{AV}$ and AA genotypes, which can react to $\mathrm{NO}$ producing $\mathrm{ONOO}^{-}$, resulting in lipoperoxidation. When $\mathrm{Hg}$ enters into the cell, $\mathrm{MeHg}$ rapidly binds to reduced glutathione (GSH) besides binding other thiol groups (-SH) present in proteins. Not only does GSH deflect mercury from binding to target proteins inside the cell, but it also serves as a means of removing mercury from the cells. As GSH is consumed through mercury binding, lower amounts of this molecule are available to prevent $\mathrm{H}_{2} \mathrm{O}_{2}$ toxic effects, which in turn (by Fenton's reaction or spontaneously) produce ${ }^{\circ} \mathrm{OH}$ that leads to DNA damage. B. AA genotype presents the highest $\mathrm{O}_{2}{ }^{-}$- dismutation rates between genotypes resulting in excessive production of $\mathrm{H}_{2} \mathrm{O}_{2}$ that needs to be processed by glutathione (mitochondria and cytoplasm) and catalase (cytoplasm). However, $\mathrm{H}_{2} \mathrm{O}_{2}$ production rates exceeding enzymatic metabolization capacity in physiological conditions, result in DNA damage. This genotype, in the presence of $\mathrm{Hg}$, is exposed to a higher amount of $\mathrm{H}_{2} \mathrm{O}_{2}$ than they are used to, leading to an increase in DNA damage, reaching the cell apoptosis/necrosis.

The study described herein presents some methodological concerns. The choice to perform a short test was based on the idea that the protocol included a large number of subjects to evaluate toxic effects in in vitro human blood samples exposed to MeHg. However, the protocol used herein did not permit the analysis of various oxidative and antioxidant biomarkers in the samples exposed or not exposed to MeHg. However, these limitations can be solved through complementary cell culture studies that search causal mechanisms to discover whether gene polymorphism has toxic response influence as observed in the present study. 
Because human studies involving controlled toxicological exposure are ethically inappropriate, studies that integrate genetic polymorphism and in vitro protocols as performed here can, despite their methodological limitations, be useful to our understanding of the toxicogenetic effects of compounds such as $\mathrm{Hg}$.

\section{ACKNOWLEDGMENTS}

Research supported by grants and fellowships of the Brazilian research support agencies Conselho Nacional de Desenvolvimento Científico e Tecnológico (\#300969/2009-0), Fundação de Amparo à Pesquisa do Estado do Rio Grande do Sul (\#1006741), and Coordenação de Aperfeiçoamento de Pessoal de Nível Superior.

\section{REFERENCES}

Ariza ME, Bijur GN and Williams MV (1998). Lead and mercury mutagenesis: role of $\mathrm{H}_{2} \mathrm{O}_{2}$, superoxide dismutase, and xanthine oxidase. Environ. Mol. Mutagen. 31: 352-361.

Beyer WF Jr and Fridovich I (1987). Effect of hydrogen peroxide on the iron-containing superoxide dismutase of Escherichia coli. Biochemistry 26: 1251-1257.

Bica CG, de Moura da Silva LL, Toscani NV, da Cruz IB, et al. (2009). MnSOD gene polymorphism association with steroid-dependent cancer. Pathol. Oncol. Res. 15: 19-24.

Bica CG, da Silva LL, Toscani NV, Zettler CG, et al. (2010). Polymorphism (ALA16VAL) correlates with regional lymph node status in breast cancer. Cancer Genet. Cytogenet. 196: 153-158.

Bussolaro D, Filipak NF and Ribeiro CA (2010). Responses of hepatocytes to DDT and methyl mercury exposure. Toxicol. In Vitro 24: 1491-1497.

Clarkson TW and Magos L (2006). The toxicology of mercury and its chemical compounds. Crit. Rev. Toxicol. 36: 609-662.

Cooper GS, Parks CG, Treadwell EL, St Clair EW, et al. (2004). Occupational risk factors for the development of systemic lupus erythematosus. J. Rheumatol. 31: 1928-1933.

Costa F, Dornelles E, Manica-Cattani MF, Algarve TD, et al. (2012). Influence of Val16Ala SOD2 polymorphism on the in-vitro effect of clomiphene citrate in oxidative metabolism. Reprod. Biomed. Online 24: 474-481.

Dickinson DA and Forman HJ (2002). Cellular glutathione and thiols metabolism. Biochem. Pharmacol. 64: 1019-1026.

do Nascimento JL, Oliveira KR, Crespo-Lopez ME, Macchi BM, et al. (2008). Methylmercury neurotoxicity \& antioxidant defenses. Indian J. Med. Res. 128: 373-382.

dos Santos Montagner GF, Sagrillo M, Machado MM, Almeida RC, et al. (2010). Toxicological effects of ultraviolet radiation on lymphocyte cells with different manganese superoxide dismutase Ala16Val polymorphism genotypes. Toxicol. In Vitro 24: 1410-1416.

Duarte MM, Moresco RN, Duarte T, Santi A, et al. (2010). Oxidative stress in hypercholesterolemia and its association with Ala16Val superoxide dismutase gene polymorphism. Clin. Biochem. 43: 1118-1123.

Engström KS, Wennberg M, Stromberg U, Bergdahl IA, et al. (2011). Evaluation of the impact of genetic polymorphisms in glutathione-related genes on the association between methylmercury or $\mathrm{n}-3$ polyunsaturated long chain fatty acids and risk of myocardial infarction: a case-control study. Environ. Health 10: 33.

Farina M, Rocha JB and Aschner M (2011). Mechanisms of methylmercury-induced neurotoxicity: evidence from experimental studies. Life Sci. 89: 555-563.

Ferreira AL and Matsubara LS (1997). Free radicals: concepts, associated diseases, defense system and oxidative stress. Rev. Assoc. Med. Bras. 43: 61-68.

Fukai T and Ushio-Fukai M (2011). Superoxide dismutases: role in redox signaling, vascular function, and diseases. Antioxid. Redox. Signal. 15: 1583-1606.

Gottlieb MG, Schwanke CH, Santos AF, Jobim PF, et al. (2005). Association among oxidized LDL levels, MnSOD, apolipoprotein E polymorphisms, and cardiovascular risk factors in a south Brazilian region population. Genet. Mol. Res. 4: 691-703.

Grotto D, Barcelos GR, Valentini J, Antunes LM, et al. (2009). Low levels of methylmercury induce DNA damage in rats: protective effects of selenium. Arch. Toxicol. 83: 249-254.

Grotto D, Valentini J, Fillion M, Passos CJ, et al. (2010). Mercury exposure and oxidative stress in communities of the 
Brazilian Amazon. Sci. Total Environ. 408: 806-811.

Gundacker C, Gencik M and Hengstschlager M (2010). The relevance of the individual genetic background for the toxicokinetics of two significant neurodevelopmental toxicants: mercury and lead. Mutat. Res. 705: 130-140.

Halliwell B and Whiteman M (2004). Measuring reactive species and oxidative damage in vivo and in cell culture: how should you do it and what do the results mean? Br. J. Pharmacol. 142: 231-255.

Havarinasab S and Hultman P (2005). Organic mercury compounds and autoimmunity. Autoimmun. Rev. 4: 270-275.

Hussain S, Atkinson A, Thompson SJ and Khan AT (1999). Accumulation of mercury and its effect on antioxidant enzymes in brain, liver, and kidneys of mice. J. Environ. Sci. Health B 34: 645-660.

Jansson G and Harms-Ringdahl M (1993). Stimulating effects of mercuric- and silver ions on the superoxide anion production in human polymorphonuclear leukocytes. Free Radic. Res. Commun. 18: 87-98.

Kakko S, Päivänsalo M, Koistinen P, Kesaniemi YA, et al. (2003). The signal sequence polymorphism of the MnSOD gene is associated with the degree of carotid atherosclerosis. Atherosclerosis 168: 147-152.

LeBel CP, Ischiropoulos H and Bondy SC (1992). Evaluation of the probe 2',7'-dichlorofluorescin as an indicator of reactive oxygen species formation and oxidative stress. Chem. Res. Toxicol. 5: 227-231.

Lund BO, Miller DM and Woods JS (1991). Mercury-induced $\mathrm{H}_{2} \mathrm{O}_{2}$ production and lipid peroxidation in vitro in rat kidney mitochondria. Biochem. Pharmacol. 42 (Suppl): S181-S187.

Montano MA, Barrio Lera JP, Gottlieb MG, Schwanke CH, et al. (2009). Association between manganese superoxide dismutase (MnSOD) gene polymorphism and elderly obesity. Mol. Cell Biochem. 328: 33-40.

Mosmann T (1983). Rapid colorimetric assay for cellular growth and survival: application to proliferation and cytotoxicity assays. J. Immunol. Methods 65: 55-63.

Moszczyński P, Rutowski J, Slowinski S and Bem S (1998). Immunological effects of occupational exposure to metallic mercury in the population of T-cells and NK-cells. Analyst 123: 99-103.

Murphy MP (2009). How mitochondria produce reactive oxygen species. Biochem. J. 417: 1-13.

Rutkowski J, Moszczyński P, Bem S and Szewczyk A (1998). Efficacy of urine determination of early renal damage markers for nephrotoxicity monitoring during occupational exposure to mercury vapours. Med. Pr. 49: 129-135.

Shanker G, Syversen T, Aschner JL and Aschner M (2005). Modulatory effect of glutathione status and antioxidants on methylmercury-induced free radical formation in primary cultures of cerebral astrocytes. Brain Res. Mol. Brain Res. 137: 11-22.

Shenker BJ, Pankoski L, Zekavat A and Shapiro IM (2002). Mercury-induced apoptosis in human lymphocytes: caspase activation is linked to redox status. Antioxid. Redox Signal. 4: 379-389.

Su L, Wang M, Yin ST, Wang HL, et al. (2008). The interaction of selenium and mercury in the accumulations and oxidative stress of rat tissues. Ecotoxicol. Environ. Saf. 70: 483-489.

Sutton A, Imbert A, Igoudjil A, Descatoire V, et al. (2005). The manganese superoxide dismutase Ala16Val dimorphism modulates both mitochondrial import and mRNA stability. Pharmacogenet. Genom. 15: 311-319.

Taufer M, Peres A, de Andrade VM, de Oliveira G, et al. (2005). Is the Val16Ala manganese superoxide dismutase polymorphism associated with the aging process? J. Gerontol. A Biol. Sci. Med. Sci. 60: 432-438.

Tian C, Fang S, Du X and Jia C (2011). Association of the C47T polymorphism in SOD2 with diabetes mellitus and diabetic microvascular complications: a meta-analysis. Diabetologia 54: 803-811.

Virtanen JK, Rissanen TH, Voutilainen S and Tuomainen TP (2007). Mercury as a risk factor for cardiovascular diseases. J. Nutr. Biochem. 18: 75-85.

Zelko IN, Mariani TJ and Folz RJ (2002). Superoxide dismutase multigene family: a comparison of the CuZn-SOD (SOD1), Mn-SOD (SOD2), and EC-SOD (SOD3) gene structures, evolution, and expression. Free Radic. Biol. Med. 33: 337-349.

Zorov DB, Juhaszova M and Sollott SJ (2006). Mitochondrial ROS-induced ROS release: an update and review. Biochim. Biophys. Acta 1757: 509-517. 